\title{
Diosgenin promotes antitumor immunity and PD-1 antibody efficacy against melanoma by regulating intestinal microbiota
}

\author{
Mengxue Dong ${ }^{1}$, Zhefeng Meng ${ }^{2}$, Kudelaidi Kuerban ${ }^{1}$, Feilong Qi ${ }^{1}$, Jiayang Liu', Yuxi Wei ${ }^{1}$, Qian Wang ${ }^{1}$, \\ Shanshan Jiang ${ }^{3}$, Meiqing Feng ${ }^{1}$ and Li Y $\mathrm{e}^{1,2}$
}

\begin{abstract}
Diosgenin, a natural steroidal saponin, can exert antitumor effect by regulating immune function and improving intestinal microbiota. The response to anti-PD-1 immunotherapy is associated with intestinal microbiota and effector $T$ cells in tumor microenvironment. We hypothesize that the modulation of diosgenin on intestinal microbiota can facilitate antitumor immunity and the therapeutic efficacy of PD-1 antibody. In melanoma-bearing C57BL/6 mice, we observed that the anti-melanoma effect of diosgenin relied more on antitumor immunity than direct tumor inhibition activity evidenced by obvious $\mathrm{CD} 4^{+} / \mathrm{CD}^{+}$T-cell infiltration and IFN- $\gamma$ expression in tumor tissues, and it could improve the compositions of intestinal microbiota. Antibiotics impaired the therapeutic efficacy and immunity responses of diosgenin through disturbing intestinal microbiota, indicating the importance of intestinal microbiota in diosgenin's in vivo antitumor activity. More importantly, the combined administration of PD-1 antibody with diosgenin aggravated the tumor necrosis and apoptosis by eliciting augmented T-cell responses. Taken together, diosgenin can be used as a microecological regulator to induce antitumor immunity and improve the efficacy of immune checkpoint antibody, making it more suitable for the treatment of malignant tumors.
\end{abstract}

\section{Introduction}

Tumor immunotherapy has aroused general concern due to its capacity to enhance the host's immune surveillance to recognize tumor cells, and then make use of autoimmune function to eradicate tumor and produce sustained antitumor immune response ${ }^{1}$. In the process of tumor progression, the immune activity of $\mathrm{T}$ cells is dampened by upregulation of the immune checkpoint molecules such as PD-1/PD-L1 and CTLA-4 in the

\footnotetext{
Correspondence: Li Ye (yelil@fudan.edu.cn)

${ }^{1}$ Department of Microbiological \& Biochemical Pharmacy, School of Pharmacy, Fudan University, 201203 Shanghai, China

${ }^{2}$ Minhang Branch, Zhongshan Hospital, Fudan University, 201199 Shanghai, China

Full list of author information is available at the end of the article.

These authors contributed equally: Mengxue Dong, Zhefeng Meng

Edited by T. Kaufmann
}

tumor microenvironment, resulting in tumor immune escape. Consequently, blockade of immune checkpoint has become a research focus of current tumor immunotherapy ${ }^{2,3}$.

Recently, immunotherapy represented by immune checkpoint inhibitors targeting the PD-1/PD-L1 axis and CTLA-4 has delivered favorable results in many solid tumors ${ }^{4}$. In advanced melanoma patients, treatment with PD-1 antibody pembrolizumab prolonged progression-free survival (PFS) and overall survival with less adverse events ${ }^{5}$. Among patients with non-small-cell lung cancer and a high tumor mutational burden, significantly longer PFS occurred with combination therapy of PD-1 antibody nivolumab and CTLA-4 antibody ipilimumab than with chemotherapy ${ }^{6}$. Besides, pembrolizumab was approved by FDA for the

\section{(c) The Author(s) 2018}

(c) (i) Open Access This article is licensed under a Creative Commons Attribution 4.0 International License, which permits use, sharing, adaptation, distribution and reproduction c. in any medium or format, as long as you give appropriate credit to the original author(s) and the source, provide a link to the Creative Commons license, and indicate if changes were made. The images or other third party material in this article are included in the article's Creative Commons license, unless indicated otherwise in a credit line to the material. If material is not included in the article's Creative Commons license and your intended use is not permitted by statutory regulation or exceeds the permitted use, you will need to obtain permission directly from the copyright holder. To view a copy of this license, visit http://creativecommons.org/licenses/by/4.0/. 

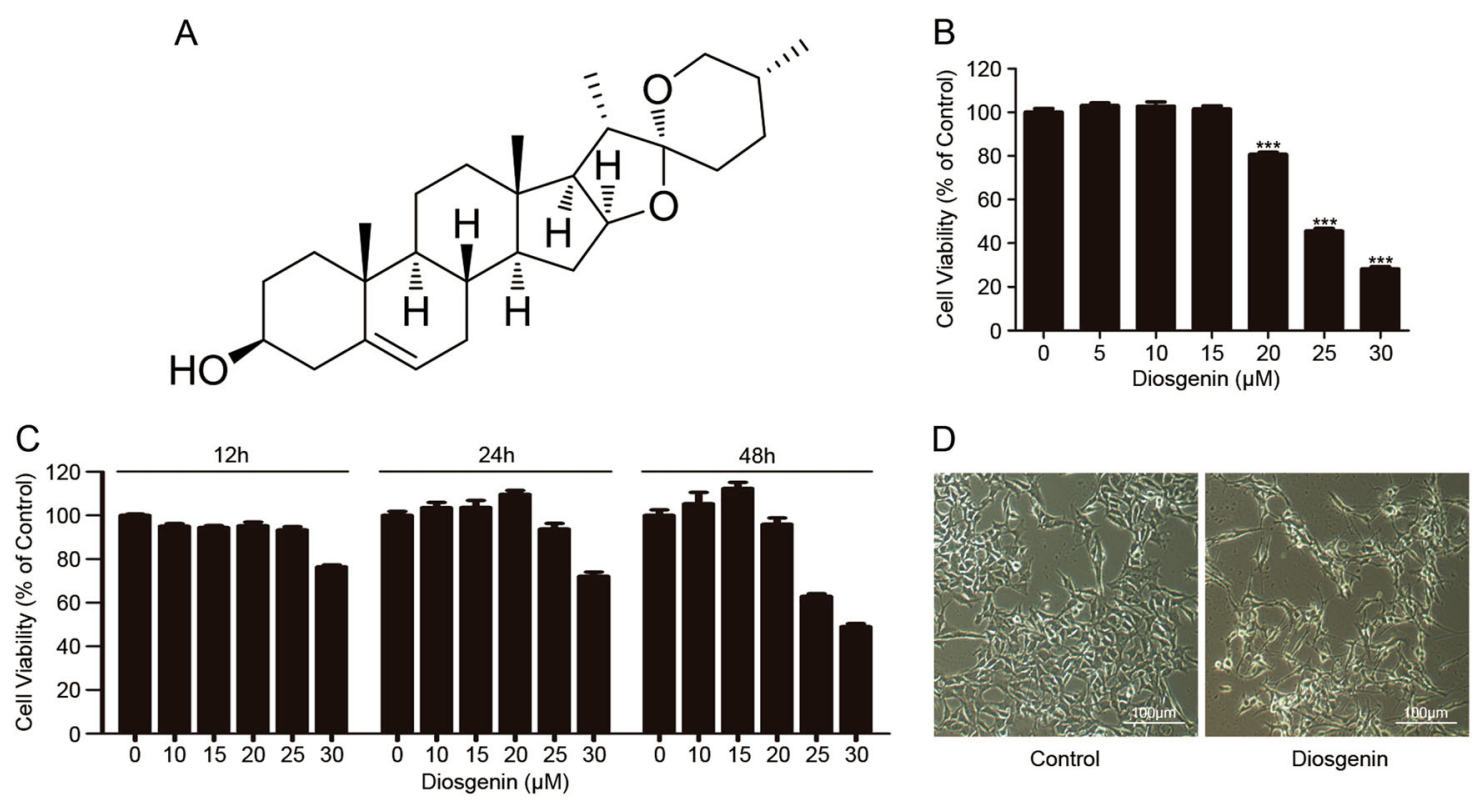

Fig. 1 Diosgenin induced cytotoxicity in B16F10 cells. a The structure of diosgenin. b B16F10 cells were treated with various concentrations of diosgenin for $48 \mathrm{~h}$, and then MTT assay was applied to measure the cell viability $\left(\mathrm{IC}_{50}\right.$ of diosgenin $\left.=25 \mu \mathrm{M}\right)$. All these data were represented as mean \pm SD $\left.{ }^{* * *} P<0.001\right)$. c B16F10 cells were exposed to different concentrations of diosgenin for indicated times. Cell viability was measured by MTT assay. d B16F10 cells were administered with $25 \mu \mathrm{M}$ diosgenin for $48 \mathrm{~h}$ and the morphological alterations of cells were observed by an inverted microscope

treatment of solid tumor with high microsatellite instability or defective mismatch repair, and became the first "broadspectrum anticancer drug" based on common biomarkers rather than specified tumor locations ${ }^{7}$. This has accelerated single-agent or multi-drug combination therapy of immune checkpoint inhibitors represented by PD-1 antibodies. Despite all of these advantages, unfortunately only about $25 \%$ of patients with solid tumors can benefit from such immunotherapy. It emphasizes that how to improve the clinical response rate of patients to immune checkpoint antibodies is the main obstacle.

It is thought that interpatient heterogeneity in the clinical response to immune checkpoint inhibitors is closely interrelated with cancer-cell-autonomous cues, tumormicroenvironmental factors, and host-related influences ${ }^{8}$. Among them, intestinal microbiota as a promoter of tumor immunotherapy has now become a topical issue ${ }^{9,10}$. Numerous researches suggest that regulating the microbiota may modulate cancer immunotherapy and promote the efficacy of immune checkpoint antibodies ${ }^{11-13}$. There are two studies that highlight the key role of the intestinal microbiota in mediating tumor response to immune checkpoint inhibitors: (1) The antitumor efficacy of CTLA-4 antibody depended on the immunostimulatory effect of intestinal microbiota, in which distinct bacterial species could promote the maturation of intratumoral dendritic cells and induced interleukin-12-dependent Th1 immune response ${ }^{14}$. (2) Intestinal Bifidobacterium could facilitate the dendritic cell function that in turn enhanced tumor-specific $\mathrm{CD}^{+} \mathrm{T}$-cell priming, and accumulation in the tumor microenvironment subsequently promoted the efficacy of PD-L1 antibody against melanoma ${ }^{11}$. Meanwhile, prior or concomitant modulation of the intestinal microbiota can optimize treatment outcomes of immune checkpoint inhibitors ${ }^{8}$. This phenomenon has been further confirmed in human cancer patients. Three recent published studies reported that intestinal commensal microbiome was closely related to the efficacy of anti-PD-1 immunotherapy in melanoma and epithelial cancer patients $^{15-17}$.

Diosgenin is a natural steroidal saponin derived from the genus Dioscorea. As a traditional Chinese medicine (TCM), it can be used for controlling hyperlipidemic, hyperglycemic, and cardiovascular diseases, and even has immunomodulatory and antitumor functions ${ }^{18-20}$. It has been reported that diosgenin and its derivatives possessed the ability to remedy melanoma, leukemia, and lung cancer by inducing cell cycle arrest, cell differentiation, and apoptosis ${ }^{21-23}$. Specifically, diosgenin can significantly inhibit the growth of sarcoma- 180 tumor by improving both specific and nonspecific cellular immune effects $^{23}$, increase the expressions of IFN- $\gamma$ and antigenspecific $\operatorname{IgG}_{2 \mathrm{a}}$, and enhance immune response mediated by Th1 cells ${ }^{24}$. It was recently found that diosgenin has 


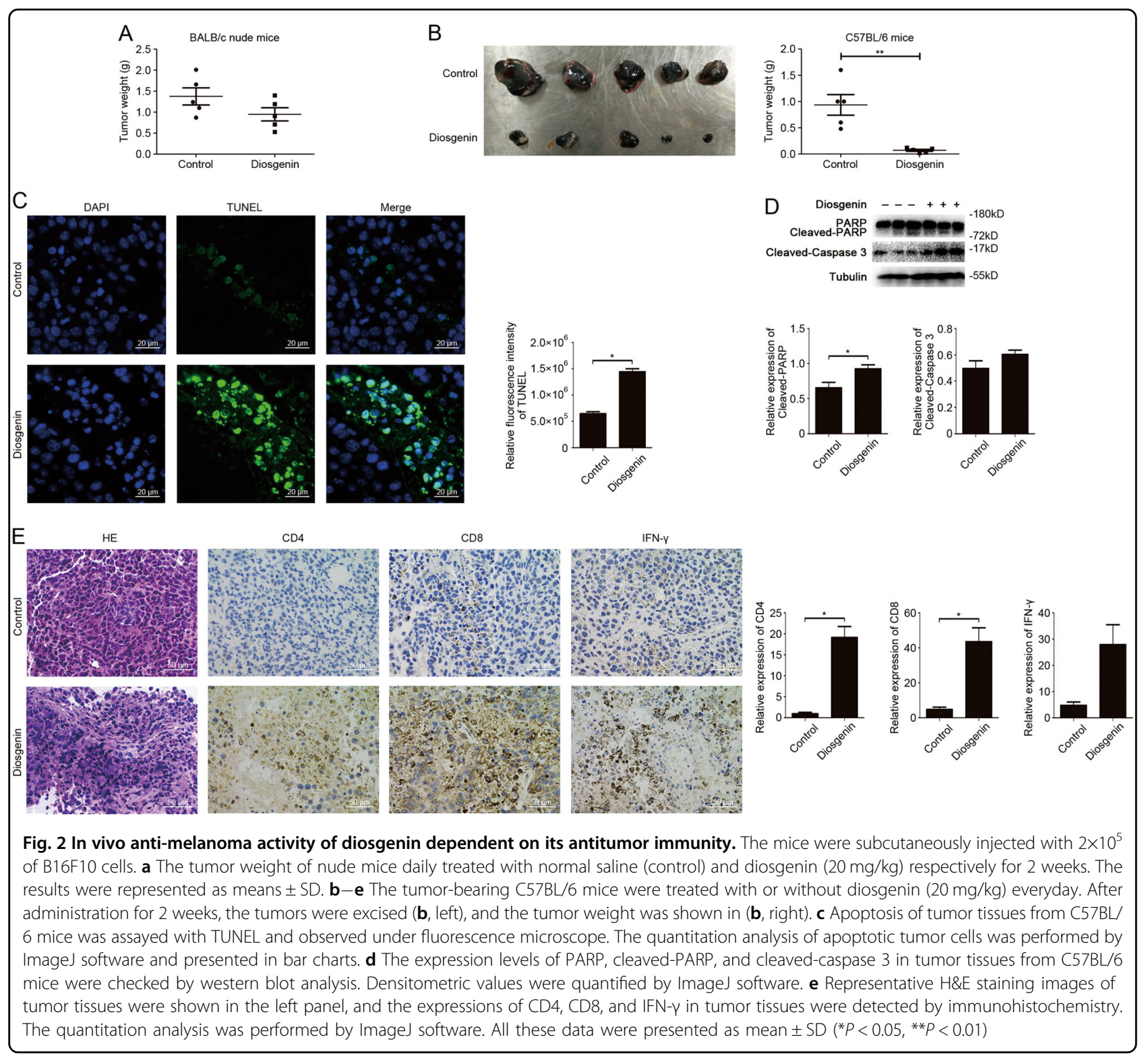

similar activity to prebiotics. Oral diosgenin can notably upregulate the composition of intestinal lactic acid bacteria in mice, which breaks the normal concept that prebiotics are mostly oligosaccharides ${ }^{25}$.

In this study, we aim to determine whether the antitumor immune effect caused by diosgenin is achieved through regulating the intestinal microbiota in melanoma-bearing immunocompetent mice. Moreover, taking into consideration that the efficacy of PD-1 antibody was influenced by intestinal microbiota and positively correlated with the number of effector $\mathrm{T}$ cells in the tumor microenvironment ${ }^{26,27}$, this study further explores whether the diosgenin-induced intestinal microbiota modulation and antitumor immunity can improve the therapeutic efficacy of PD-1 antibody.

\section{Results}

\section{Diosgenin exerted anti-melanoma activity in vitro}

The structure of diosgenin is shown in Fig. 1a. 3-(4, 5dimethylthiazol-2-yl)-2, 5-diphenyltetrazolium bromide (MTT) assay was used to determine the cytotoxicity of diosgenin by detecting the cell viability. After exposure to different concentrations of diosgenin for indicated times, the B16F10 melanoma cell viability decreased in a dose- and time-dependent manner within the tested range (Fig. 1b, c). Meanwhile, cells displayed visible morphological changes after treatment with diosgenin, including cell shrink, fragmentation, and reduced number of cells (Fig. 1d).

Altogether, diosgenin induced cell viability reduction and obvious morphological alteration in melanoma cells, which indicated the anti-melanoma effect of diosgenin in vitro. 


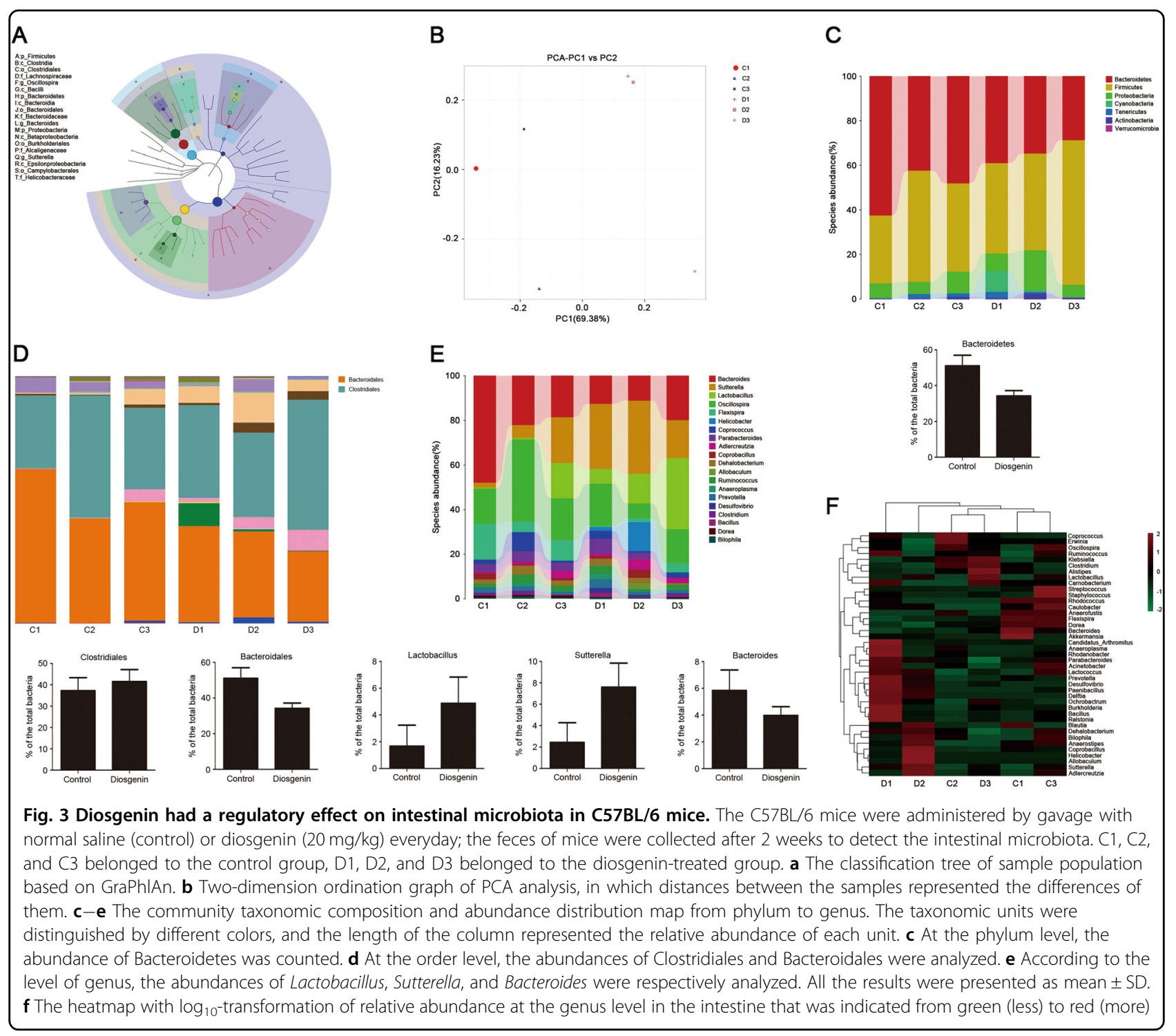

In vivo anti-melanoma effect of diosgenin was associated with antitumor immunity

In the mice model of subcutaneous tumor produced by B16F10 melanoma cells, the antitumor effect was initially evaluated after diosgenin administration by measuring the tumor weight. As shown in Fig. 2a, diosgenin slowed down the tumor growth, but the inhibition rate was only $31.1 \%$ as compared to the control in $\mathrm{BALB} / \mathrm{c}$ nude mice. The inhibition rate was calculated as $[(A-B) / A] \times 100 \%$, where $A$ and $B$ were the average tumor weights of the negative control and experimental groups respectively. Surprisingly, the tumor weight reduced more obviously in diosgenintreated immunocompetent C57BL/6 mice with inhibition rate of $92.5 \%$ (Fig. 2b). TUNEL assay displayed a significantly large number of apoptotic cells in tumor tissues harvested from C57BL/6 mice treated with diosgenin (Fig. 2c). Western blot analysis also illustrated that the cleavage of apoptosis-related proteins such as PARP and caspase-3 were both upregulated (Fig. 2d), indicating that diosgenin can induce more apoptosis in tumor tissues. Moreover, hematein-eosin (H\&E) staining detected extensive necrosis within the tumor in diosgenin-treated cohort (Fig. 2e). Because of the remarkable difference in anti-melanoma effects of diosgenin between immunodeficient mice and immunocompetent mice, we speculated that diosgenin might induce antitumor immunity besides direct tumor inhibition activity in vivo. Immunohistochemical analysis of tumor tissues was then conducted to explore whether diosgenin can trigger immune effect in tumor-bearing C57BL/6 mice. Compared with the control, the expressions of CD4, CD8, and IFN- $\gamma$ were positive in diosgenin-treated cohort, suggesting the infiltration of 


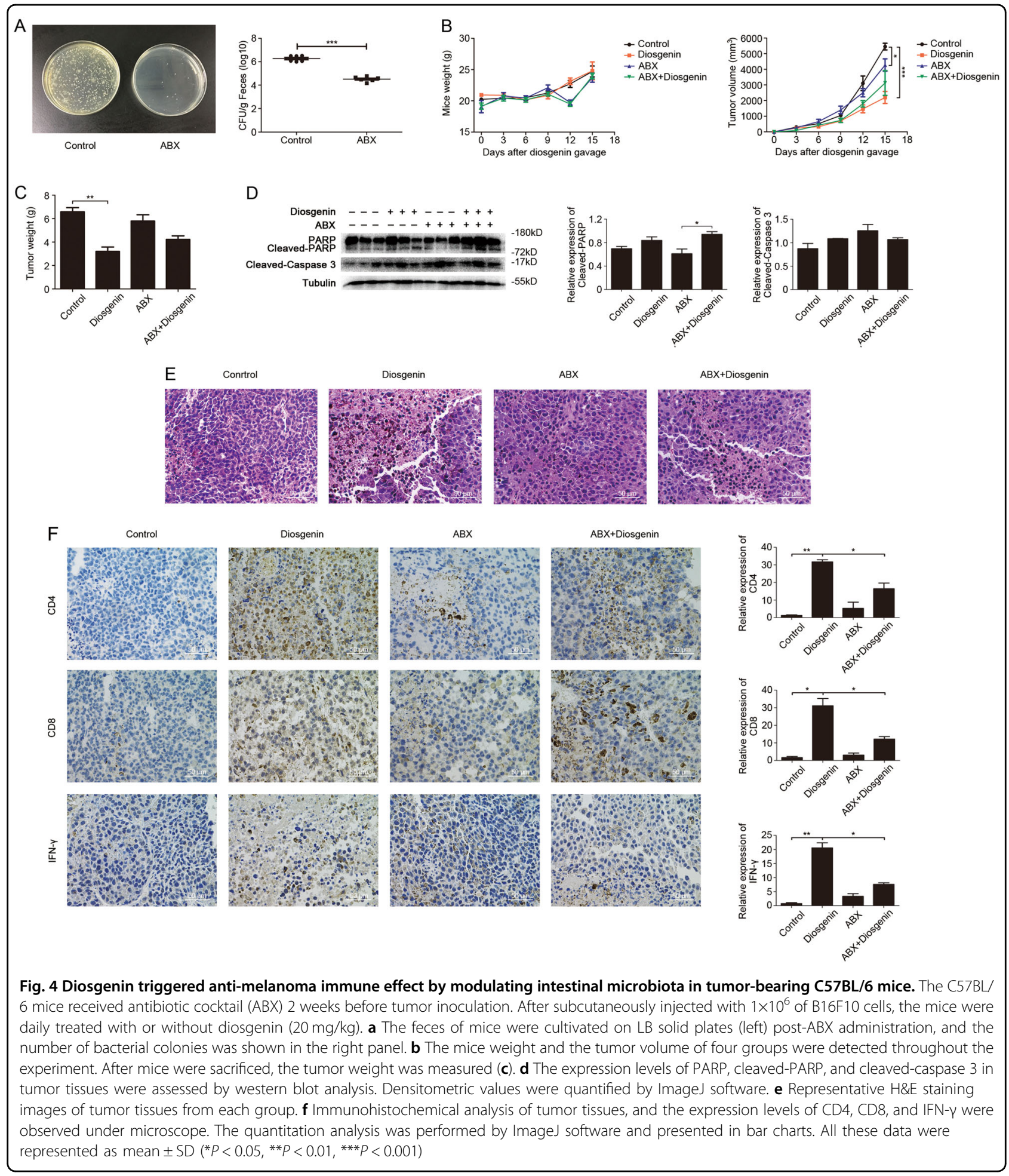

$\mathrm{CD}^{+} \mathrm{T}$ and $\mathrm{CD}^{+} \mathrm{T}$ cells as well as augmented immune response in tumor tissues (Fig. 2e).

These results demonstrated that in vivo anti-melanoma effect of diosgenin relied more on antitumor immune effect than direct tumor inhibition activity.
The modulation of intestinal microbiota was triggered by diosgenin

Relative abundance of each Operational Taxonomic Unit (OTU) and other taxonomic levels (from phylum to genus) was evaluated for each sample. The number of 
sequences clustered within each OTU (or other taxonomic levels) was converted to a fraction representing the relative contribution of each feature to each individual. The classification tree showed the hierarchical relationship among all taxonomic units (represented by nodes) from phylum to genus (from inner ring to outer ring) in the sample population, and the taxons of the top 20 relative abundances were identified by letters (Fig. 3a). Principal component analysis (PCA) maximized the distances between the samples in the coordinate system to reflect the actual differences. As shown in Fig. 3b, the intestinal microbial community structure notably changed after diosgenin administration. In the community taxonomic composition and abundance distribution map at phylum level, the column of Bacteroidetes was shorter than that in the control after treatment with diosgenin, indicating that Bacteroidetes were downregulated (Fig. 3c). Meanwhile, based on bacterial order composition, the increase of Clostridiales and the reduction of Bacteroidales were observed (Fig. 3d). It can also be found that Lactobacillus and Sutterella were obviously upregulated as well as Bacteroides were downregulated at genus level (Fig. 3e). For heatmap, $\log _{10}$-transformation was applied on the relative abundance data matrix, which allowed visualizing similarities or differences between samples. After exposure to diosgenin, microbial community differences became smaller, making the species composition have the higher degree of uniformity (Fig. 3f).

In summary, these results illustrated that diosgenin, as a modulator, could change the composition of the intestinal microbiota in C57BL/6 mice.

\section{Diosgenin induced anti-melanoma activity and immune effect by regulating intestinal microbiota}

To explore whether the intestinal microbiota affects the antitumor immune effect of diosgenin in vivo, C57BL/6 mice received antibiotic cocktail (ABX), and the varieties and numbers of their intestinal microbiota were visibly changed after 2 weeks of this treatment (Fig. 4a). In subcutaneous melanoma models, the mice weight increased steadily in the control and diosgenintreated cohorts, and the weight of the mice after antibiotic treatment tended to increase with slight fluctuations, indicating that the diosgenin and $\mathrm{ABX}$ were safe at the administration dosage. Meanwhile, among the four groups the tumor volume in diosgenin-treated cohort increased most slowly from day 9 till the remaining experimental period (Fig. 4b). When mice were sacrificed on day 15 and the tumors were resected, the tumor weight of cohort treated with diosgenin was $3203.33 \pm 641.43 \mathrm{mg}$ versus $6586.67 \pm 603.52 \mathrm{mg}$ of the control, and the tumor weight of the cohort cotreated with diosgenin and ABX was $4220.00 \pm$
$540.65 \mathrm{mg}$ (Fig. 4c). Afterwards, western blot analysis was performed on harvested tumor tissues to analyze apoptosis. As shown in Fig. 4d, the cohort treated with diosgenin as well as cohort cotreated with diosgenin and $A B X$ both increased the cleavage of PARP and caspase-3, but there were no significant differences between these two. Histological and immunohistochemical analysis displayed that, in tumor-bearing mice administered with diosgenin, the tumor necrosis and $\mathrm{CD} 4^{+} / \mathrm{CD}^{+} \mathrm{T}$-cell infiltration increased along with an enhancement of IFN- $\gamma$ expression in tumor tissues. However, ABX treatment could greatly cut down all of these growth trends mentioned above (Fig. 4e, f).

All of these results indicated that the integrity of the intestinal microbiota was crucial for diosgenin to induce the antitumor activity and immune effect in vivo.

\section{Diosgenin enhanced the efficacy of PD-1 antibody by eliciting immune effect}

The experiments above confirmed that diosgenin could regulate intestinal microbiota and induce immune effect, and it is reported that the efficacy of PD-1 antibody had a certain correlation with both of them. Thus, we further evaluated whether diosgenin could enhance the response rate and the efficacy of PD- 1 antibody. B16F10 subcutaneous tumor model was established, and then the C57BL/6 mice were treated with PD-1 mAb 6 days later. As shown in Fig. 5a, the mice weight continued to increase steadily throughout the experiment in all cohorts, and the tumor volume increased more slowly from day 9 till the remaining experimental period in the cohort cotreated with diosgenin and PD-1 mAb as well as the cohort treated with diosgenin or PD-1 mAb alone than the control. Then, mice were sacrificed and tumors were resected after 15 days of treatment. Mean tumor weight of the cohort cotreated with diosgenin and PD- $1 \mathrm{mAb}$ was $1980.00 \pm 861.22 \mathrm{mg}$ versus 3203.33 $\pm 641.43 \mathrm{mg}$ of the cohort treated with diosgenin alone, and tumor weight of the cohort treated with PD-1 mAb alone was $2530.00 \pm 584.04 \mathrm{mg}$ (Fig. 5b). Western blot analysis showed that combined administration of diosgenin and PD-1 mAb increased the cleavage of PARP and caspase-3, indicating that apoptosis was enhanced in tumor cells (Fig. 5c). After planned administration, the number of tumor-infiltrating $\mathrm{CD}^{+}$and $\mathrm{CD} 8^{+}$ $\mathrm{T}$ cells increased from 22 to $30.8 \%$ and from 4.8 to $8.6 \%$ respectively in diosgenin-treated cohort, while that increased to 27.6 and $17.5 \%$ respectively in combined administration cohort (Fig. 5d). Histological and immunohistochemical analysis further revealed that in tumor-bearing mice treated with diosgenin and PD-1 $\mathrm{mAb}$, the tumor necrosis, $\mathrm{CD} 4^{+} / \mathrm{CD}^{+} \mathrm{T}$-cell infiltration, and IFN- $\gamma$ expression in tumor tissues were all 


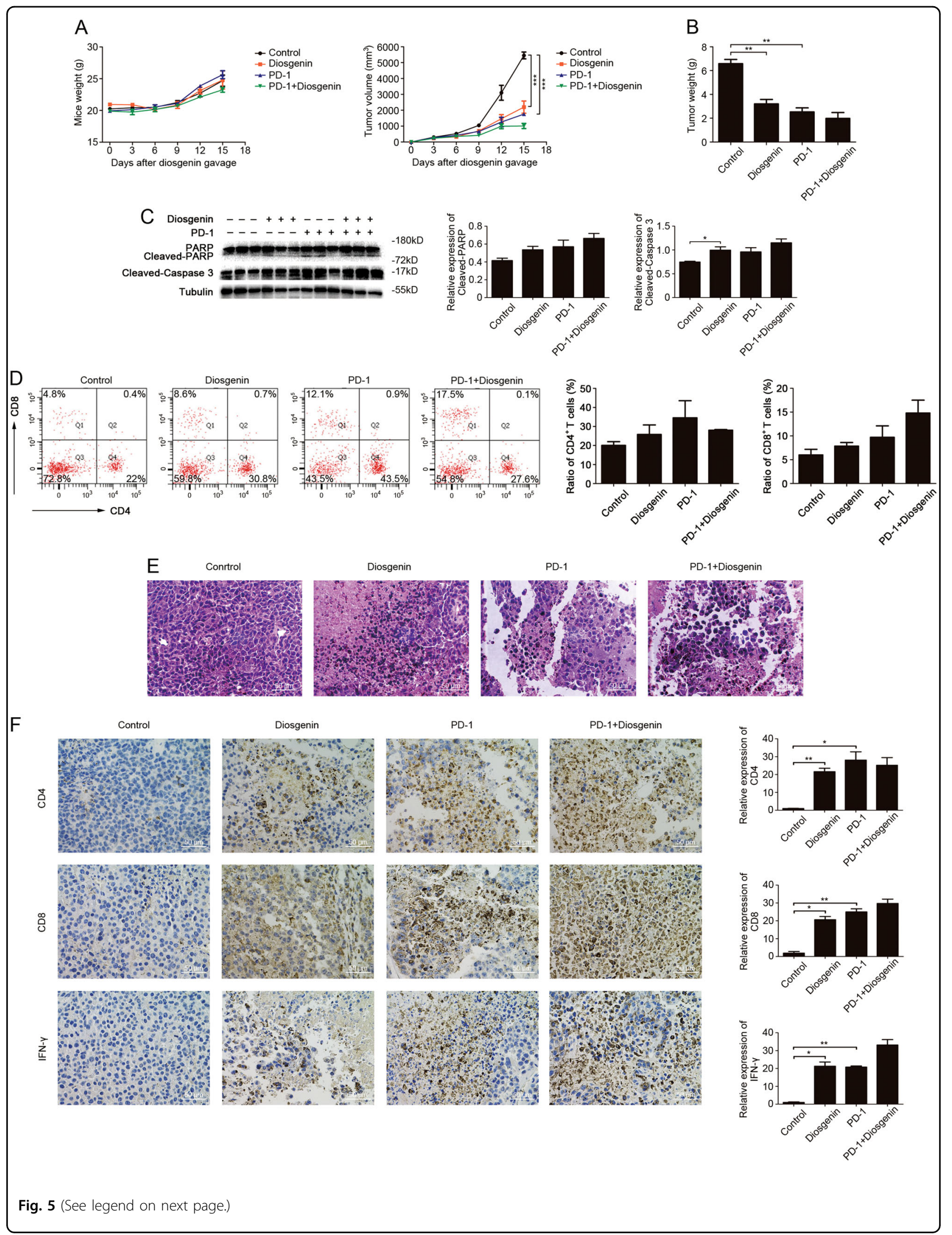


(see figure on previous page)

Fig. 5 Combined administration of diosgenin and PD-1 mAb achieved the best antitumor effect. After subcutaneously injected with $1 \times 10^{6}$ of B16F10 cells, the C57BL/ 6 mice were daily treated with or without diosgenin $(20 \mathrm{mg} / \mathrm{kg})$, and then injected intraperitoneally with PD-1 mAb on days 6,9 , and 12 post-tumor implantation. a The mice weight and the tumor volume of four groups were detected throughout the experiment. $\mathbf{b}$ After the whole experiment, the tumor weight was measured. c The expression levels of PARP, cleaved-PARP, and cleaved-caspase 3 in tumor tissues were assessed by western blot analysis. Densitometric values were quantified by ImageJ software. $\mathbf{d}$ The number of $\mathrm{CD} 4^{+} / \mathrm{CD} 8^{+} \mathrm{T}$ cells in tumor tissues was analyzed by flow cytometry, and the percentage of these cells was presented in bar charts as mean $\pm \mathrm{SD}$. e Representative H\&E staining images of tumor tissues from each group. f The expression levels of CD4, CD8, and IFN- $\gamma$ in tumor tissues were determined by immunohistochemistry, and the quantitation analysis was performed by ImageJ software. All these data were presented as mean $\pm \mathrm{SD}\left({ }^{*} P<0.05,{ }^{* *} P<0.01,{ }^{* * *} P<0.001\right)$. PD-1 indicates PD-1 monoclonal antibody

upregulated more obviously than other cohorts (Fig. 5e, f).

Collectively, these results suggested that diosgenin could improve the efficacy of PD-1 antibody by inducing immune effect.

\section{Discussion}

In recent years, plant saponins from diverse sources have attracted much attention as immune adjuvants ${ }^{28}$. The saponin components from Acacia concinna can effectively induce the humoral and cellular immune responses by enhancing the proliferation of lymphocyte and activation of effector $\mathrm{T}$ cells ${ }^{29}$. Platycodin D2 can dramatically increase the expressions of IL- 2 and IFN- $\gamma$, and then activate the Th1 immunoreaction ${ }^{30}$. The semisynthetic saponin derivative GPI-0100 can activate Th1 immunity and IFN- $\gamma$ expression in preclinical trials ${ }^{31}$. Diosgenin is a main component of TCM Di'ao xinxuekang that is mainly used for the treatment of coronary heart disease and angina, and its antitumor activity and ability to cause specific or nonspecific cellular immune effects have been demonstrated ${ }^{20,23}$.

In the present study, we first observed that diosgenin could inhibit the growth of B16F10 melanoma cells in vitro and in vivo as well as alter morphological features of tumor cells. However, its tumor suppression effect in immunocompetent C57BL/6 mice was superior to that in $\mathrm{BALB} / \mathrm{c}$ nude mice. It meant that diosgenin not only directly exerted antitumor activity, but also was likely to enhance tumor killing effect by inducing the antitumor immunity of mice. Our experiments proved that $\mathrm{CD}_{4}^{+} /$ $\mathrm{CD}^{+}{ }^{-}$T-cell infiltration and IFN- $\gamma$ expression in tumor tissues of C57BL/6 mice significantly increased after diosgenin treatment, indicating the activation of the immune system ${ }^{32}$. Simultaneously, diosgenin could trigger visible necrosis and apoptosis in tumor tissues from C57BL/6 mice. Therefore, we conclude that diosgenin's in vivo anti-melanoma activity mainly depends on its antitumor immunity induction effect.

The concept that diosgenin has similar activity to prebiotics was further confirmed by its ability to improve the intestinal microecology of Type 2 diabetic mice ${ }^{33}$.
Consequently, we investigated the diversities and the structural features of intestinal microbiota in C57BL/6 mice based on the sequencing results on $\mathrm{V} 4$ region of $16 \mathrm{~S}$ rDNA. After diosgenin administration, the abundances of the Lactobacillus genus in the Firmicutes phylum and the Sutterella genus in the Proteobacteria phylum were significantly upregulated, whereas the abundance of the Bacteroides genus in the Bacteroidetes phylum was downregulated. It has been reported that intestinal probiotics represented by Lactobacillus can inhibit tumor formation, and the metastatic melanoma patients with high abundance of Lactobacillus were sensitive to the treatment of PD-1 antibody ${ }^{17,34}$. Sutterella were positively associated with proinflammatory immune effects ${ }^{35}$. Bacteroides can induce regulatory $\mathrm{T}$ cells, its downregulation may imply the elimination of immunosuppression ${ }^{36}$. Besides, we found that diosgenin could increase the abundance of Clostridiales order and reduce the abundance of Bacteroidales order. In melanoma patients, the high abundance of intestinal Clostridiales represented better sensitivity to PD-1 antibody treatment, and the high abundance of Bacteroidales showed insensitivity to this therapy ${ }^{16}$. These results suggest that diosgenin can modulate the intestinal microbiota in C57BL/6 mice, and the diversities and the overall structural changes of intestinal microbiota triggered by diosgenin may be related to its antitumor immune effect.

Recent clinical investigation confirmed that antibiotics could induce intestinal microbiota dysbiosis and then affect antitumor immune responses and effectiveness of immune checkpoint inhibitors ${ }^{37}$. To further verify the necessity of intestinal microbiota on the antitumor immunity triggered by diosgenin, we used the antibiotic cocktail method to disturb the intestinal microbiota of C57BL/6 mice, and then observed the therapeutic efficacy of diosgenin on B16F10 melanoma ${ }^{14}$. It displayed that ABX destroyed the intestinal microbiota, which subsequently weakened the tumor suppression activity of diosgenin evidenced by increased tumor weight and growth speed. Diosgenin-induced tumor necrosis was also greatly alleviated by ABX cotreatment. Similar downtrend of $\mathrm{CD}^{+} / \mathrm{CD}^{+} \mathrm{T}$-cell infiltration and IFN- $\gamma$ expression in 


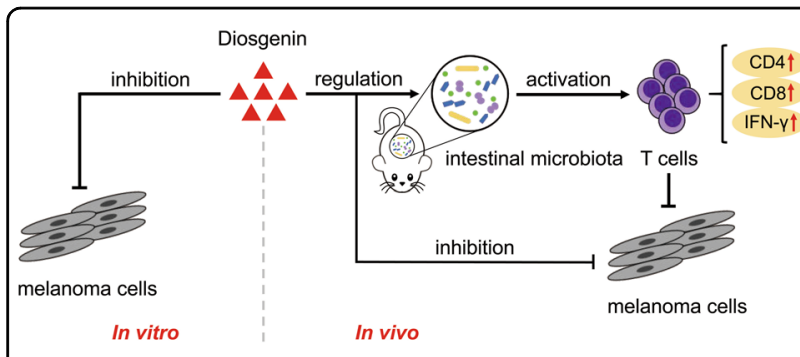

Fig. 6 Overview of anti-melanoma effect induced by diosgenin in vitro and in vivo. Diosgenin can inhibit the growth of B16F10 melanoma cells in vitro. In vivo, the anti-melanoma effect of diosgenin is mainly dependent on antitumor immunity which is induced by its regulation of intestinal microbiota in C57BL/6 mice

tumor tissues of $\mathrm{ABX}$ cotreated mice was detected by immunohistochemical analysis. These results indicate that the damage of intestinal microbiota triggered by $\mathrm{ABX}$ indeed impaired the antitumor immunity and the therapeutic efficacy of diosgenin in vivo, which confirmed our hypothesis that diosgenin-induced antitumor immune effect was mediated by the intestinal microbiota. Nevertheless, ABX treatment had little effect on the apoptosis of tumor cells induced by diosgenin, and the group treated with $\mathrm{ABX}$ alone showed a weak $\mathrm{CD} 4^{+} / \mathrm{CD}^{+} \mathrm{T}$-cell infiltration. It has been reported that the $\mathrm{ABX}$-treated mice had relatively higher frequency of effector $\mathrm{T}$ cells in gut wall or spleen and higher circulating level of IFN- $\gamma$ along with lower IL-10 level to increase the susceptibility to inflammation ${ }^{38,39}$. Thus, it is better to apply germ-free mice to confirm the role of intestinal microbiota in the antitumor effect of diosgenin in further studies.

Moreover, intestinal microbiota can improve therapeutic efficacy of $\mathrm{CpG}$ oligonucleotides, platinum compounds, and cyclophosphamide by regulating the function of some tumor-microenvironmental immune cells and facilitating tumor infiltration of effector $\mathrm{T}$ cells, which ensures a longer PFS for the patients with advanced lung and ovarian cancers ${ }^{12,13,40}$. Blockade of immune checkpoint can strengthen and maintain endogenous antitumor effect, keeping the tumor under sustained control $^{41}$. The clinical activity of PD-1/PD-L1 antibodies is positively correlated with the integrity of intestinal microbiota and the number of effector $\mathrm{T}$ cells in the tumor microenvironment, and modulation of intestinal microbiota composition may be a promising strategy to improve clinical benefit from PD-1/PD-L1 antibodies ${ }^{27,37,42}$. So, we further explored whether diosgenin could promote the therapeutic efficacy of PD-1 antibody by regulating intestinal microbiota to induce antitumor immunity in C57BL/6 mice. Our experiments proved that the combined administration of diosgenin and PD-1 mAb enhanced the tumor growth inhibition as well as aggravated the tumor necrosis and apoptosis. We also found that the $\mathrm{CD}^{+} / \mathrm{CD}^{+}$T-cell infiltration and IFN- $\gamma$ expression in tumor tissues were distinctly upregulated through this combination treatment. These results illustrate that diosgenin can improve the response rate and the therapeutic efficacy of PD-1 antibody by eliciting immune effect.

In conclusion, our research proves that diosgenin can trigger growth inhibition of B16F10 melanoma cells in vitro as well as tumor necrosis, apoptosis, and activation of antitumor immunity in vivo. Meanwhile, diosgenin has the regulatory effect on intestinal microbiota in mice, and its antitumor immune effect is associated with intestinal microbiota (Fig. 6). Furthermore, diosgenin can enhance the therapeutic efficacy of PD-1 antibody by eliciting $\mathrm{T}$-cell immune effect. This is the first report to study the effects of diosgenin on antitumor immunity and the therapeutic efficacy of immune checkpoint antibody based on the intestinal microecology. These results have important implications for the application of TCM in tumor immunotherapy and combination therapy with immune checkpoint inhibitors.

\section{Materials and methods \\ Regents and antibodies}

Diosgenin with a purity of more than $98 \%$ was obtained from Nanjing Spring \& Autumn Biotech Co., Ltd (Jiangsu, China). Other reagents were purchased as follows: MTT (Sigma-Aldrich), InVivoMAb anti-mouse PD-1 (clone 29F.1A12, BioXCell), vancomycin, ampicillin, neomycin, and metronidazole (Dalian Meilun Biotech Co., Ltd), RIPA Lysis Buffer and phenylmethanesulfonyl fluoride (PMSF) (Beyotime Biotechnology), xylene and $\mathrm{H}_{2} \mathrm{O}_{2}$ (Sinopharm Chemical Reagent Co., Ltd.), 10* Proteinase $\mathrm{K}$ stock solution and hematoxylin (Google Biotechnology), Diaminobenzidine (DAB) Developer (DAKO), EDTA (PH9.0) Antigen Repair Buffer (Servicebio), TUNEL Assay kit (Roche), MoBio PowerSoil $^{\circledast}$ DNA Isolation Kit (MoBio), Quant-iT PicoGreen dsDNA Assay Kit (Invitrogen), TruSeq Nano DNA LT Library Prep Kit and MiSeq Reagent Kit V3 (Illumina). The primary antibodies including CD4, $\mathrm{CD} 8$, and IFN- $\gamma$ as well as the horseradish peroxidase (HRP)-conjugated secondary antibody against rabbit for immunohistochemistry were purchased from Servicebio, Inc (Boston, USA). Anti-mouse antibodies CD4 (clone GK1.5) and CD8a (clone 53-6.7) were purchased from BioLegend, and CD3e monoclonal antibody (clone 145-2C11) was purchased from eBioscience for flow cytometry analysis.

\section{Cell lines and culture conditions}

The C57BL/6-derived melanoma cell line B16F10, purchased from Cell Bank of Chinese Academy of 
Sciences, Shanghai Branch (Shanghai, China), was cultured in Dulbecco's Modified Eagle Medium (DMEM) (Corning) containing 10\% fetal bovine serum (Gibco), $100 \mathrm{IU} / \mathrm{mL}$ of penicillin and $100 \mu \mathrm{g} / \mathrm{mL}$ of streptomycin (Beyotime Institute of Biotechnology) at $37^{\circ} \mathrm{C}$ in a humidified atmosphere of $5 \% \mathrm{CO}_{2}$ incubator.

\section{Cytotoxicity on B16F10 cells in vitro}

About $1 \times 10^{4}$ cells were planted in 96-well plates and allowed to attach overnight, then exposed to different concentrations of diosgenin for indicated times. Then, the incubation of these cells with MTT $(0.5 \mathrm{mg} / \mathrm{mL})$ was implemented at $37^{\circ} \mathrm{C}$ and $100 \mu \mathrm{L}$ Dimethyl Sulfoxide (DMSO) was added to dissolve the formazan crystals. The optical density was measured at the wavelength of $570 \mathrm{~nm}$ by microplate reader.

\section{Microscopy and photography}

About $5 \times 10^{3}$ cells were seeded into six-well plates and then incubated with indicated concentration of diosgenin. Cells were observed by using an inverted microscope (Nikon, Japan) after incubation for $48 \mathrm{~h}$.

\section{Animals and tumor models}

All animal experiments were carried out in compliance with the standards approved by Animal Ethical Committee of School of Pharmacy at Fudan University. The 6week-old female SPF BALB/c nude mice and C57BL/6 mice $(18-20 \mathrm{~g})$ were subcutaneously injected with appropriate density of B16F10 cells suspended in normal saline to establish tumor models.

\section{Diosgenin administration and PD-1 mAb immunotherapy in vivo}

The well-established mice were randomly divided into the indicated groups, in which the mice were daily treated with diosgenin $(20 \mathrm{mg} / \mathrm{kg}, 0.2 \mathrm{~mL})$ by gavage on the second day post-tumor implantation, and normal saline treatment was served as the control. For combination therapy experiments, mice were additionally injected intraperitoneally with $200 \mu \mathrm{g}$ PD- $1 \mathrm{mAb}$ in $300 \mu \mathrm{L}$ PBS on days 6,9 , and 12 post-tumor implantation. After 15 days of this administration, the mice were sacrificed, and the solid tumors were excised and weighed.

\section{Antibiotic cocktail}

The mice were treated with antibiotics 2 weeks before tumor implantation and continued until the end of the experiment. A mixture of vancomycin $(500 \mathrm{mg} / \mathrm{L})$, ampicillin $(1 \mathrm{~g} / \mathrm{L})$, neomycin $(1 \mathrm{~g} / \mathrm{L})$, and metronidazole $(1 \mathrm{~g} / \mathrm{L})$ were added in sterile drinking water. Solutions were changed three times a week, and care was taken to avoid light. Antibiotic activity was analyzed by cultivating the fecal pellets of the mice resuspended in normal saline on $\mathrm{LB}$ solid culture plates for $24 \mathrm{~h}$ at $37^{\circ} \mathrm{C}$ with $5 \% \mathrm{CO}_{2}$ for aerobic conditions.

\section{Western blotting}

The isolated tumor tissues were homogenized after adding the appropriate amount of RIPA Lysis Buffer and PMSF, and then centrifuged $\left(12,000 \times g, 4{ }^{\circ} \mathrm{C}\right)$ for $10 \mathrm{~min}$ to obtain the supernatant. Equal amounts of lysate proteins $(20 \mu \mathrm{g})$ were electrophoretically separated by sodium dodecyl sulfate-polyacrylamide gel electrophoresis and transferred to polyvinylidene fluoride membranes. Membranes were blocked in Tris-buffered saline with Tween 20 (TBST) supplemented with 5\% bovine serum albumin (BSA) at room temperature for $2 \mathrm{~h}$ and incubated at $4{ }^{\circ} \mathrm{C}$ overnight with primary antibodies. They were then subjected to HRP-conjugated secondary antibodies at room temperature for $1.5 \mathrm{~h}$ and proteins were detected using enhanced chemiluminescence system (Pierce, Rockford, IL, USA). Intensities in the resulting bands were quantified by ImageJ software.

\section{TUNEL assay}

The isolated tumor tissues were fixed with $4 \%$ paraformaldehyde for at least $24 \mathrm{~h}$, embedded in paraffin and sectioned. Then, paraffin sections were subjected to deparaffinization, rehydration, and permeabilization with proteinase $\mathrm{K}$ for $30 \mathrm{~min}$. After being washed three times with PBS, the tumor tissue sections were treated with TUNEL Assay kit following the manufacturer' s instruction. Samples were counterstained with hematoxylin after DAB coloration, dehydrated with xylene and then mounted. The apoptosis of tumor cells was visualized by fluorescence microscopy (Nikon, Japan).

\section{Histology and immunohistochemistry}

Histological observation of tumor tissues was applied to examine the tumor necrosis. Paraffin sections of tumor tissues were deparaffinized and rehydrated, and then stained routinely with Harris hematoxylin and eosin.

For immunohistochemistry, the antigen of tumor paraffin sections was retrieved in EDTA $(\mathrm{PH}=9)$ Antigen Repair Buffer. To quench the endogenous peroxidase activity, the samples were incubated with $3 \% \mathrm{H}_{2} \mathrm{O}_{2}$ for $30 \mathrm{~min}$ and followed by blocking in 3\% BSA in PBS. The slides were covered with correlative primary antibodies and incubated at $4{ }^{\circ} \mathrm{C}$ overnight, followed by incubation with secondary antibody at room temperature for $50 \mathrm{~min}$. Then, the tissue sections were counterstained with hematoxylin after DAB coloration and mounted. All the samples were detected by an inverted microscope (Nikon, Japan). 


\section{Flow cytometry}

After the planned treatment, the isolated tumor tissues were filtered through $70 \mu \mathrm{m}$ cell strainer (BD Falcon, USA) to obtain the single cells. The harvested cells were washed twice with PBS, and then stained in the dark with anti-mouse antibodies for CD3e, CD4, and CD8a at $4{ }^{\circ} \mathrm{C}$ for $30 \mathrm{~min}$. Analysis was performed immediately by using an FACS Calibur flow cytometer (Becton-Dickinson, Fullerton, CA, USA).

\section{Bacterial identification in fecal samples}

The C57BL/6 mice, randomized into two groups, were treated with diosgenin $(20 \mathrm{mg} / \mathrm{kg})$ and normal saline (control) by gavage everyday respectively. After 2 weeks of this administration, the feces of mice were collected and frozen at $-80^{\circ} \mathrm{C}$.

Bacterial DNA from the feces of mice was extracted using MoBio PowerSoil ${ }^{\circledR}$ DNA Isolation Kit and subjected to PCR amplification. The highly variable V4 region of the bacterial 16S rRNA (about $280 \mathrm{bp}$ ) was used for sequencing to analyze microbial diversity. The PCR products were quantified on Microplate reader (BioTek, FLx800) by using Quant-iT PicoGreen dsDNA Assay Kit, and then mixed according to the amount of data required for each sample. Thereafter, the DNA library was constructed by using Illumina's TruSeq Nano DNA LT Library Prep Kit. After quality control, sequencing, and quantitation of the library, MiSeq Reagent Kit V3 (600 cycles) was used for double-end sequencing of $2 \times 300 \mathrm{bp}$ on the MiSeq machine.

According to the quality of the sequences, the preliminary screening was conducted on the original sequencing data. The high-quality sequences were clustered into OTUs at $97 \%$ of identity by using QIIME software. Representative sequences of each OTU were used for taxonomic identification and phylogenetic analysis. Based on the abundance distribution of OTUs in different samples, the diversity of each sample was assessed. Subsequently, the specific composition of each sample at different classification levels was analyzed.

\section{Statistical analysis}

The statistical analysis was performed by GraphPad Prism 5 with one-way ANOVA or two tailed Student' $s$ $t$ test. All data were presented as mean \pm SD and values of $P<0.05$ were considered to be statistically remarkable.

\section{Acknowledgements}

This study was supported by National Natural Science Foundation of China (No. 81572979), Shanghai Biomedical Science and Technology Support Project (No. 18431902800) and National Key Basic Research Program of China (2015CB931800)

\section{Author details}

${ }^{1}$ Department of Microbiological \& Biochemical Pharmacy, School of Pharmacy, Fudan University, 201203 Shanghai, China. ${ }^{2}$ Minhang Branch, Zhongshan
Hospital, Fudan University, 201199 Shanghai, China. ${ }^{3}$ Department of Pharmacy, Shanghai Ninth People's Hospital, Shanghai Jiao Tong University School of Medicine, 200011 Shanghai, China

Conflict of interest

The authors declare that they have no conflict of interest.

Publisher's note

Springer Nature remains neutral with regard to jurisdictional claims in published maps and institutional affiliations.

Received: 12 June 2018 Revised: 12 August 2018 Accepted: 14 September 2018

Published online: 10 October 2018

\section{References}

1. Wolchok, J. D. \& Chan, T. A. Cancer: antitumour immunity gets a boost. Nature 515, 496-498 (2014).

2. Sharma, P. \& Allison, J. P. The future of immune checkpoint therapy. Science 348, 56-61 (2015).

3. Weiner, G. J. Building better monoclonal antibody-based therapeutics. Nat. Rev. Cancer 15, 361-370 (2015).

4. Chen, L. \& Han, X. Anti-PD-1/PD-L1 therapy of human cancer: past, present, and future. J. Clin. Invest. 125, 3384-3391 (2015).

5. Robert, C. et al. Pembrolizumab versus ipilimumab in advanced melanoma. $N$. Engl. J. Med. 372, 2521-2532 (2015).

6. Hellmann, M. D. et al. Nivolumab plus ipilimumab in lung cancer with a high tumor mutational burden. N. Engl. J. Med. 378, 2093-2104 (2018).

7. Yu, Y. Molecular classification and precision therapy of cancer: immune checkpoint inhibitors. Front. Med. 12, 1-7 (2018).

8. Pitt, J. M. et al. Resistance mechanisms to immune-checkpoint blockade in cancer: tumor-intrinsic and -extrinsic factors. Immunity 44, 1255-1269 (2016).

9. Leslie, M. MICROBIOME microbes aid cancer drugs. Science 350, 614-615 (2015).

10. Erdman, S. E. Gut microbiota: microbes offer engineering strategies to combat cancer. Nat. Rev. Gastroenterol. Hepatol. 13, 125-126 (2016).

11. Sivan, A. et al. Commensal Bifidobacterium promotes antitumor immunity and facilitates anti-PD-L1 efficacy. Science 350, 1084-1089 (2015).

12. lida, N. et al. Commensal bacteria control cancer response to therapy by modulating the tumor microenvironment. Science 342, 967-970 (2013).

13. Viaud, S. et al. The intestinal microbiota modulates the anticancer immune effects of cyclophosphamide. Science 342, 971-976 (2013).

14. Vetizou, M. et al. Anticancer immunotherapy by CTLA-4 blockade relies on the gut microbiota. Science 350, 1079-1084 (2015).

15. Routy, B. et al. Gut microbiome influences efficacy of PD-1-based immunotherapy against epithelial tumors. Science 359, 91-97 (2018).

16. Gopalakrishnan, V. et al. Gut microbiome modulates response to anti-PD-1 immunotherapy in melanoma patients. Science 359, 97-103 (2018).

17. Matson, $\mathrm{V}$. et al. The commensal microbiome is associated with anti-PD-1 efficacy in metastatic melanoma patients. Science 359, 104 (2018).

18. Ghezali, L., Liagre, B., Limami, Y., Beneytout, J. L. \& Leger, D. Y. Sonic hedgehog activation is implicated in diosgenin-induced megakaryocytic differentiation of human erythroleukemia cells. PLOS ONE 9, e95016 (2014).

19. He, Z . et al. Diosgenin inhibits the migration of human breast cancer MDA-MB-231 cells by suppressing Vav2 activity. Phytomedicine 21, 871-876 (2014).

20. He, Z. et al. Anti-tumour and immunomodulating activities of diosgenin, a naturally occurring steroidal saponin. Nat. Prod. Res. 26, 2243-2246 (2012).

21. Jiang, S. et al. Diosgenin induces ROS-dependent autophagy and cytotoxicity via mTOR signaling pathway in chronic myeloid leukemia cells. Phytomedicine 23, 243-252 (2016).

22. Kim, D. S., Jeon, B. K., Lee, Y. E., Woo, W. H. \& Mun, Y. J. Diosgenin induces apoptosis in HepG2 cells through generation of reactive oxygen species and mitochondrial pathway. Evid-Based Compl. Alt. 2, 981675 (2012).

23. Raju, J. \& Mehta, R. Cancer chemopreventive and therapeutic effects of diosgenin, a food saponin. Nutr. Cancer 61, 27-35 (2009). 
24. Jan, T. R., Wey, S. P., Kuan, C. C., Liao, M. H. \& Wu, H. Y. Diosgenin, a steroidal sapogenin, enhances antigen-specific lgG2a and interferon-gamma expression in ovalbumin-sensitized BALB/C mice. Planta Med. 73, 421-426 (2007).

25. Huang, C. H., Cheng, J. Y., Deng, M. C., Chou, C. H. \& Jan, T. R. Prebiotic effect of diosgenin, an immunoactive steroidal sapogenin of the Chinese yam. Food Chem. 132, 428-432 (2012).

26. Tang, $H$. et al. Facilitating $T$ cell infiltration in tumor microenvironment overcomes resistance to PD-L1 blockade. Cancer Cell. 30, 500 (2016).

27. Ribas, A. et al. PD-1 blockade expands intratumoral memory T cells. Cancer Immunol. Res 4, 194-203 (2016).

28. Sun, H. X., Ye, Y. P., Pan, H. J. \& Pan, Y. J. Adjuvant effect of Panax notoginseng saponins on the immune responses to ovalbumin in mice. Vaccine $\mathbf{2 2}$ 3882-3889 (2004).

29. Kukhetpitakwong, R. et al. Immunological adjuvant activities of saponin extracts from the pods of Acacia concinna. Int. Immunopharmacol. 6 1729-1735 (2006).

30. Xie, Y., Deng, W., Sun, H. \& Li, D. Platycodin D2 is a potential less hemolytic saponin adjuvant eliciting Th1 and Th2 immune responses. Int. Immunopharmacol. 8, 1143-1150 (2008).

31. Liu, H. et al. Preclinical evaluation of the saponin derivative GPI-0100 as an immunostimulating and dose-sparing adjuvant for pandemic influenza vaccines. Vaccine 29, 2037-2043 (2011).

32. Llosa, N. J. et al. The vigorous immune microenvironment of microsatellite instable colon cancer is balanced by multiple counter-inhibitory checkpoints. Cancer Discov. 5, 43-51 (2015).
33. Yan, $\mathrm{H}$. et al. Intake of total saponins and polysaccharides from Polygonatum kingianum affects the gut microbiota in diabetic rats. Phytomedicine $\mathbf{2 6}, \mathbf{4 5 - 5 4}$ (2017).

34. Erdman, S. E. \& Poutahidis, T. Gut bacteria and cancer. Biochim. Biophys. Acto 1856, 86-90 (2015).

35. Chen, C. C.. Chen, K. J., Kong, M. S., Chang, H. J. \& Huang, J. L. Alterations in the gut microbiotas of children with food sensitization in early life. Pediatr. Allergy Immunol. 27, 254-262 (2016).

36. Smith, P. M. et al. The microbial metabolites, short-chain fatty acids, regulate colonic Treg cell homeostasis. Science 341, 569-573 (2013).

37. Derosa, L. et al. Negative association of antibiotics on clinical activity of immune checkpoint inhibitors in patients with advanced renal cell and nonsmall-cell lung cancer. Ann. Oncol. 29, 1437-1444 (2018).

38. Gulden, E. et al. Microbiota control immune regulation in humanized mice. $J C$ Insight 2, e91709 (2017)

39. Ekmekciu, I. et al. Immune responses to broad-spectrum antibiotic treatment and fecal microbiota transplantation in mice. Front. Immunol. 8, 397 (2017).

40. Daillere, R. et al. Enterococcus hirae and Barnesiella intestinihominis facilitate cyclophosphamide-induced therapeutic immunomodulatory effects. Immunity 45, 931-943 (2016).

41. Baumeister, S. H., Freeman, G. J., Dranoff, G. \& Sharpe, A. H. Coinhibitory pathways in immunotherapy for cancer. Annu. Rev. Immunol. 34, 539-573 (2016).

42. Herbst, R. S. et al. Predictive correlates of response to the anti-PD-L1 antibody MPDL3280A in cancer patients. Nature 515, 563-567 (2014). 